九州大学学術情報リポジトリ

Kyushu University Institutional Repository

\title{
Completeness of Extended Unification Based on Basic Narrowing
}

Yamamoto, Akihiro

Department of Information Systems Interdisciplinary Graduate School of Engineering Sciences Kyushu University

http://hdl. handle. net/2324/3108

出版情報: RIFIS Technical Report. 2, 1988-03-19. Research Institute of Fundamental Information Science, Kyushu University

バージョン :

権利関係 : 
RIFIS-TR-CS-2

\title{
RIFIS Technical Report
}

Completeness of Extended Unification Based on Basic Narrowing

\author{
Akihiro Yamamoto
}

March 19, 1988

Research Institute of Fundamental Information Science

Kyushu University 33

Fukuoka 812, Japan 


\title{
Completeness of Extended Unification Based on Basic Narrowing
}

\author{
Akihiro YAMAMOTO \\ Department of Information Systems \\ Interdisciplinary Graduate School of Engineering Sciences \\ Kyushu University 39 \\ 6-1, Kasuga-koen, Kasuga, Fukuoka, 816 JAPAN
}

Mailing Address: Research Institute of Fundamental Information Science

Kyushu University 33, Fukuoka, 812 JAPAN

\begin{abstract}
In this paper we prove the completeness of unification based on the basic narrowing. First we prove the completeness of unification based on original narrowing under a weaker condition than the previous proof. Then we discuss the relation between basic narrowing and innermost reduction as the lifting lemma, and prove the completeness of unification based on the basic narrowing. Moreover, we give the switching lemma to combine the previous algorithm and our new proof.
\end{abstract}

\section{Introduction}

The main contribution of this paper is a proof that the extended unification procedure based on basic narrowing is complete under some conditions. The procedure was first given by Hullot [2], and then improved by Bosco et al. [1]. The improved procedure [1] was obtained by translating every basic narrowing sequence into an SLD-refutation sequence. It is now very important as a foundation of logic-functional programming languages, but the proof of its completeness is not straightforward.

Hullot [2] proved the lifting lemma for basic narrowing as that of SLD-resolution. However, his proof is incomplete since we found a counter example for Lemma 3 [2]. To prove the lifting lemma for basic narrowing, we need to construct a basic reduction from every term, but its existence is not guaranteed in general. By clarifying the function of innermost reduction, we overcome this difficulty and show the existence.

We first show, under a weaker condition than [2], the completeness of the unification based on original narrowing. That is, the term rewriting system is simply assumed weakly canonical. Then we establish the relation between innermost reduction and basic narrowing as the lifting lemma, and prove the completeness of the unification based on basic narrowing. Thus we suppose the condition that the term rewriting system is both weakly canonical and normalizing with innermost reduction. Moreover, we show the relation between the completeness theorem and the algorithm in [1] by clarifying the procedure which searches an innermost redex. Thus the relation is stated as the switching lemma.

The paper is organized as follows. In the next section, we explain the narrowing and basic narrowing. In Section 3, we prove the completeness of unification based on narrowing under a weaker condition than that 
in [2], and discuss the problem in the case of basic narrowing. In Section 4, we prove the completeness of unification based on basic narrowing under a weaker condition than that in [2]. In Section 5, we discuss the relation of our proof and the algorithm given in [1] as a form of the switching lemma.

\section{Narrowing and Basic Narrowing.}

In the present paper we treat the extended unification in a first order equality theory $E$ using a term rewriting system $R$. An $E$-unifier of two terms $t$ and $s$ is a substitution $\theta$ such that $E \vDash s \theta=t \theta$, where $\vDash$ represents the logical consequence and the predicate symbol $=$ is interpreted as the equality. We consider the case that $E$ is given as a reflexive symmetric transitive closure of the reduction relation on $R$, which is defined below.

The symbol $\equiv$ denotes the syntactical identity. The set of all variables occurring in a term $t$ is denoted by $V(t)$. We prepare the special function symbol $e q$ only for the use of the description of procedures.

We assume that the term rewriting system $R=\left\{\alpha_{i} \rightarrow \beta_{i} ; i \in I\right\}$ satisfies the following conditions:

(i) $V\left(\alpha_{i}\right) \supset V\left(\beta_{i}\right)$,

(ii) $\alpha_{i}$ is not a variable,

(iii) $I$ is finite.

We denote the occurrence of every subterm of a term $t$ by a sequence of positive integers as follows:

(i) The occurrence of $t$ is the empty sequence (denoted by $\lambda$ ),

(ii) If the occurrence of the subterm $f\left(t_{1}, \ldots, t_{n}\right)$ is $u$, then the occurrence of the term $t_{i}$ is $u . i$.

Let $t$ be a term. Then the symbol $O c(t)$ denotes the set of occurrences of all subterms of $t$, and $O g(t)$ denotes the set of occurrences of all non-variable subterms of $t$. When $u \in O c(t), t / u$ denotes the subterm of $t$ whose occurrence is $u$, and $t[u \Leftarrow s]$ denotes the term which is obtained by replacing subword $s / u$ by a term $s$. A subterm of $t$ whose occurrence is not $\lambda$ is called a proper subterm of $t$.

Let $u$ and $v$ be two occurrences. Then we write $u \preceq v$ if there is a sequence $w$ such that $u . w=v$.

Definition 1. Let $t$ and $s$ be terms. Then $t$ is reduced to $s$ if there exist $u \in O s(t), \alpha \rightarrow \beta \in R$, and a substitution $\theta$ such that $t / u \equiv \alpha \theta$ and $s \equiv t[u \Leftarrow \beta \theta]$. When $t$ is reduced to $s$, we write $t \rightarrow s$, or $t \rightarrow[u, \alpha \rightarrow \beta, \theta] s$ to give full details. We call $t / u$ a redex of $t$.

When we regard $\rightarrow$ as a binary relation on the set of terms, we call it the reduction relation on $R$.

A term $t$ is in normal form if there exists no term $\mathrm{s}$ such that $t \rightarrow s$. If there exists a finite reduction sequence

$$
t \equiv t_{0} \rightarrow t_{1} \rightarrow \cdots \rightarrow t_{n} \equiv s
$$

such that $s$ is in normal form, then $s$ is called a normal form of $t$ and denoted by $N(t)$. Moreover, let $\theta$ be a substitution $\left\{X_{1} \leftarrow t_{1}, \ldots, X_{n} \leftarrow t_{n}\right\}$. If each of $t_{1}, \ldots, t_{n}$ is in normal form, then $\theta$ is called in normal form. For every substitution $\theta$, the substitution $\left\{X_{1} \leftarrow N\left(t_{1}\right), \ldots, X_{n} \leftarrow N\left(t_{n}\right)\right\}$, if exists, is called a normal form of $\theta$.

Definition 2. Let $t$ and $s$ be terms. Then $t$ is narrowed to $s$, if there exist $u \in O g(t), \alpha \rightarrow \beta \in R$, and a substitution $\sigma$ such that $\sigma$ is an mgu of $t / u$ and $\alpha$ and $s \equiv(t[u \Leftarrow \beta]) \sigma$. When $t$ is narrowed to $s$, we write $t \Rightarrow s$, or $t \Rightarrow_{[u, \alpha \rightarrow \beta, \sigma]} s$ to give full details. 
We assume that for every narrowing sequence

$$
t_{0} \Rightarrow_{\left[u_{0}, \alpha_{0} \rightarrow \beta_{0}, \sigma_{0}\right]} t_{1} \Rightarrow_{\left[u_{1}, \alpha_{1} \rightarrow \beta_{1}, \sigma_{1}\right]} t_{2} \Rightarrow \cdots \Rightarrow_{\left[u_{n-1}, \alpha_{n-1} \rightarrow \beta_{n-1}, \sigma_{n-1}\right]} t_{n},
$$

the variables in every rule $\alpha_{i} \rightarrow \beta_{i}$ are standardized apart ([3]). We also assume that mgu's are computed by the algorithm in $[3,4]$. Then the $E$-unification of two terms $t$ and $s$ with narrowing is to computing the set

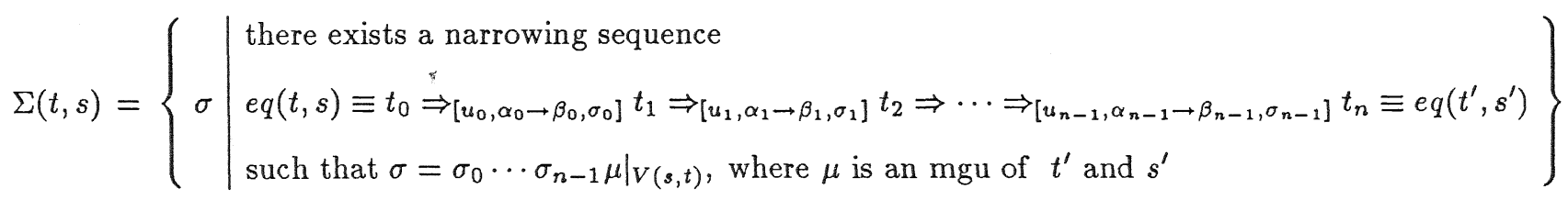
where $\left.\theta\right|_{V}$ denotes the restriction of a substitution $\theta$ to a set of variables $V$.

For two substitutions $\theta$ and $\tau$, we write $\theta={ }_{E} \tau$ if $E \vDash X \theta=X \tau$ for every variable $X$.

Definition 3. The $E$-unification algorithm based on narrowing is complete if for every two terms $t$ and $s$ $\Sigma(t, s)$ is a complete set of $E$-unifiers of $t$ and $s$, i.e., for every $E$-unifier $\theta$ of $t$ and $s$ there exist $\sigma \in \Sigma(t, s)$ and a substitution $\gamma$ such that $\left.\theta\right|_{V(s) \cup V(t)}=\left.{ }_{E} \sigma \gamma\right|_{V(s) \cup V(t)}$.

The basic narrowing is introduced in order to improve the $E$-unification algorithm based on narrowing.

Definition 4. A narrowing sequence

$$
t_{0} \Rightarrow_{\left[u_{0}, \alpha_{0} \rightarrow \beta_{0}, \sigma_{0}\right]} t_{1} \Rightarrow_{\left[u_{1}, \alpha_{1} \rightarrow \beta_{1}, \sigma_{1}\right]} t_{2} \Rightarrow \cdots \Rightarrow_{\left[u_{n-1}, \alpha_{n-1} \rightarrow \beta_{n-1}, \sigma_{n-1}\right]} t_{n}
$$

is basic if there exists a sequence of sets of occurrences $U_{0}, \ldots, U_{n}$ which satisfies the following conditions:

(B-1) $U_{0}=O g\left(t_{0}\right)$

(B-2) $u_{i} \in U_{i} \subset O c\left(t_{i}\right) \quad(0 \leq i \leq n-1) \quad$ and $\quad U_{n} \subset O c\left(t_{n}\right)$,

(B-3) $\quad U_{i+1}=\left(U_{i}-\left\{v \in U_{i} ; u_{i} \preceq v\right\}\right) \cup\left\{u_{i} . v ; v \in O g\left(\beta_{i}\right)\right\} \quad(0 \leq i \leq n-1)$.

Example 1. Let $R=\{f(X, b) \rightarrow g(X), a \rightarrow b\}$. Then a narrowing sequence

$$
f(a, X) \Rightarrow f(b, X) \Rightarrow g(b),
$$

is basic because the sequence

$$
U_{0}=\{\lambda, 1\}, U_{1}=\{\lambda, 1\}, U_{2}=\{\lambda\}
$$

satisfies the conditions (B-1), (B-2), and (B-3). However,

$$
f(a, X) \Rightarrow g(a) \Rightarrow g(b)
$$

is not basic because $U_{0}$ and $U_{1}$ must be $\{\lambda, 1\}$ and $\{\lambda\}$ respectively so that $U_{0}$ satisfies (B-1), (B-2) and (B-3), but $U_{1}$ violates (B-2).

The $E$-unification of two terms $t$ and $s$ with basic narrowing is to computing the set

$\Sigma_{B}(t, s)=\left\{\begin{array}{l}\sigma \\ \begin{array}{l}\text { there exists a basic narrowing sequence } \\ e q(t, s) \equiv t_{0} \Rightarrow_{\left[u_{0}, \alpha_{0} \rightarrow \beta_{0}, \sigma_{0}\right]} t_{1} \Rightarrow_{\left[u_{1}, \alpha_{1} \rightarrow \beta_{1}, \sigma_{1}\right]} t_{2} \Rightarrow \cdots \Rightarrow_{\left[u_{n-1}, \alpha_{n-1} \rightarrow \beta_{n-1}, \sigma_{n-1}\right]} t_{n} \equiv e q\left(t^{\prime}, s^{\prime}\right) \\ \text { such that } \sigma=\left.\sigma_{0} \cdots \sigma_{n-1} \mu\right|_{V(s, t)}, \text { where } \mu \text { is an mgu of } t^{\prime} \text { and } s^{\prime}\end{array}\end{array}\right\}$ as in the case of narrowing. 
Definition 5. The $E$-unification algorithm based on basic narrowing is complete if for every two terms $t$ and $s \Sigma(t, s)$ is a complete set of $E$-unifiers.

[1] has given an efficient algorithm for $E$-unification based on basic narrowing as explained below.

Definition 6. Let $U$ be a set of occurrences of subterms of $t$. An occurrence $u$ in is innermost in $U$ if there is no occurrence $v$ in $U$ such that $u \preceq v$ and $v \neq u$.

Algorithm 1 (Bosco, Giovannetti and Moiso [1]).

unify $(t, s, S) \quad /^{*} t$ and $s$ are terms and $S$ is a rule selecting an innermost occurrence from $B * /$

$E:=e q(t, s), B:=O g(e q(t, s))-\{\lambda\}$, and $\Theta:=\epsilon$

$/^{*} E$ is a term, $B$ is a set of occurrences, and $\Theta$ is a substitution */

LOOP:

if $B=\phi$ then

$\Theta:=\Theta \sigma$ and return $\Theta$ where $\sigma$ is an mgu of $t^{\prime}$ and $s^{\prime}$ where $E \equiv e q\left(t^{\prime}, s^{\prime}\right)$

else

select an innermost occurrence $u$ from $B$ according to $S$,

nondeterministically execute either (1-1) or (1-2), and goto LOOP:

(1-1) $B:=B-\{u\}$

(1-2) select nondeterministically $\alpha \rightarrow \beta$ from $R$ such that $E / u$ and $\alpha$ are unifiable, $E:=(E[u \Leftarrow \beta]) \sigma, \Theta:=\Theta \sigma$, and $B:=(B-\{u\}) \cup\{u . v ; v \in O g(\beta)\}$

where $\sigma$ is an mgu of $E / u$ and $\alpha$

end if

The search space of basic narrowing is decreased in Algorithm 1 by introducing the computation rule $S$ (or the selection rule in [1]), which selects one of the innermost occurrences from the set $B$. In a basic narrowing sequence, the terms introduced by an mgu may not be selected afterwards. Algorithm 1 satisfies the conditions of basic narrowing sequence by selecting a innermost occurrence from $B$. 


\section{The Problem on Basic Narrowing}

In this section we point out the problem on the completeness of $E$-unification based on basic narrowing. For the purpose of the discussion, we extend the completeness of $E$-unification based on narrowing. It was proved that $E$-unification based on narrowing is complete if $R$ is canonical, that is, confluent and finitely terminating ([2] Theorem 2).

Definition 7. $R$ is weakly canonical if for every term $t$ there exists only one normal form $N(t)$ and for every pair of $t$ and $s$

$$
E \vDash t=s \Leftrightarrow N(t) \equiv N(s)
$$

The following theorem is the lifting lemma for narrowing proved in [2].

Theorem 1 (Hullot [2]). Let $t$ and $s$ be terms such that $t \equiv s \eta$ for some normal substitution $\eta$. Then for a reduction sequence

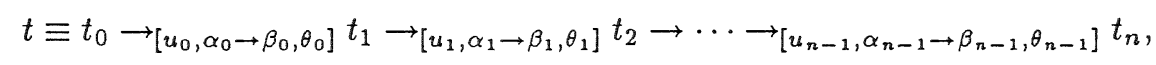

there exists a narrowing sequence

$$
s \equiv s_{0} \Rightarrow\left[u_{0}, \alpha_{0} \rightarrow \beta_{0}, \sigma_{0}\right] s_{1} \Rightarrow_{\left[u_{1}, \alpha_{1} \rightarrow \beta_{1}, \sigma_{1}\right]} s_{2} \Rightarrow \cdots \Rightarrow_{\left[u_{n-1}, \alpha_{n-1} \rightarrow \beta_{n-1}, \sigma_{n-1}\right]} s_{n},
$$

and a sequence of normal substitutions $\eta_{0}, \ldots, \eta_{n}$ such that

$$
\begin{aligned}
\eta_{0} & =\eta,\left.\quad \eta\right|_{V(s)}=\left.\sigma_{0} \cdots \sigma_{i-1} \eta_{i}\right|_{V(s)} \quad(1 \leq i \leq n) \\
t_{i} & =s_{i} \eta_{i} \quad(0 \leq i \leq n) .
\end{aligned}
$$

The assumption of Theorem 1 is only that $\eta$ is in normal form. Therefore the following theorem can be also proved in the same way as in [2].

Theorem 2 (Extension of Hullot's Theorem). If $R$ is weakly canonical, then $\Sigma(t, s)$ is a complete set of $E$-unifiers of $t$ and $s$.

Proof. Let $\theta$ be an $E$-unifier of $t$ and $s$. Because $R$ is weakly canonical, we can let $\eta$ be a normal form of $\theta$. Also we can construct a reduction sequence

$$
e q(t, s) \eta \equiv t_{0} \rightarrow t_{1} \rightarrow \cdots \rightarrow t_{n} \equiv e q(N(t \eta), N(s \eta))
$$

such that $N(t \eta) \equiv N(s \eta)$. Thus by Theorem 1 there is a narrowing sequence

$$
e q(t, s) \equiv s_{0} \Rightarrow s_{1} \Rightarrow \cdots \Rightarrow s_{n} \equiv e q\left(t^{\prime}, s^{\prime}\right)
$$

Then $t^{\prime}$ and $s^{\prime}$ are unifiable because there is a substitution $\eta_{n}$ such that $t^{\prime} \eta_{n} \equiv s^{\prime} \eta_{n}$.

To show that the above theorem is a proper extension of Hullot's Theorem, we give an example which shows the difference between canonicity and weak canonicity. 
Example 2. Let $R=\{$ repeat $\rightarrow$ repeat, repeat $\rightarrow$ halt $\}$. Then $R$ is weakly canonical. For every term $t$, its unique normal form is a term given by replacing the term repeat with halt. Moreover, we can decide $E \models s=t$ by comparing $N(s)$ and $N(t)$. However, $R$ is not finitely terminating, so $R$ is not canonical.

Now our problem is to prove the completeness of the unification based on basic narrowing. A similar result to Theorem 1 is needed for the basic narrowing. Thus we introduce the basic reduction.

Definition 8. Let $t$ and $s$ be terms such that $t \equiv s \theta$ for some substitution $\theta$. Then a reduction sequence

$$
t \equiv t_{0} \rightarrow\left[u_{0}, \alpha_{0} \rightarrow \beta_{0}, \theta_{0}\right] t_{1} \rightarrow\left[u_{1}, \alpha_{1} \rightarrow \beta_{1}, \theta_{1}\right] t_{2} \rightarrow \cdots \rightarrow\left[u_{n-1}, \alpha_{n-1} \rightarrow \beta_{n-1}, \theta_{n-1}\right] t_{n}
$$

is based on $O g(s)$ if there exists a sequence of sets of occurrences $U_{0}, \ldots, U_{n}$ which satisfies the conditions (B-2) and (B-3) in Definition 4 and the following condition (B-1') instead of (B-1):

$\left(\mathrm{B}-1^{\prime}\right) \quad U_{0}=O g(s)$.

Example 3. Let $R=\{f(X, b) \rightarrow g(X), a \rightarrow b\}$. In the same way as Example 1 we can show that a reduction sequence

$$
f(a, b) \rightarrow f(b, b) \rightarrow g(b)
$$

is based on $O g(t)$, but is not

$$
f(a, b) \rightarrow g(a) \rightarrow g(b)
$$

To get a theorem corresponding to Theorem 1, it is asserted in [2] (Lemma 3) that

if $s$ and $t$ have the relation $t \equiv s \eta$ for some normalized substitution $\eta$, then every reduction sequence from $s$ is based on $O g(s)$.

However the second sequence in Example 3 does not satisfy the above assertion. Therefore the proof of the completeness of $E$-unification based on basic narrowing does not work. Algorithm 1 is also incomplete because the proof of the completeness of Algorithm 1 in [1] is again due to the above assertion.

Algorithm 1 is found by using the one-to-one corresponding between SLD-refutation and basic narrowing sequence in [1]. The efficiency of SLD-resolution is due to the computation rule ([3]). Algorithm 1 uses not only the computation rule but also innermost occurrences. In the first sequence of Example 3, the innermost redex is only selected. On the other hand, in the second one, $f(a, b)$ is selected and it is not innermost redex. This selection makes a counter example for Lemma 3 [2]. In general, when we construct a basic reduction sequence from a term $t$ to $N(t)$, the terms introduced by the substitution at each reduction need to be in normal form because they may not be selected as redexes. From these consideration, we suggest that the innermost occurrence should essentially owe to the completeness of unification based on basic narrowing.

In the following section, we prove the completeness of unification based on basic narrowing along the consideration above. 


\section{Completeness of Unification Based on Basic Narrowing}

In the context of term rewriting systems, the word 'innermost' is not used as the meaning defined in Definition 6.

Definition 9. Suppose that $t \rightarrow[u, \alpha \rightarrow \beta, \theta]$ for some term $s$. Then $t / u$ is innermost redex and the reduction is called innermost reduction if any proper subterm of $t / u$ is normal.

Example 4. Let $R=\{f(X, b) \rightarrow g(X), a \rightarrow b\}$. Then the innermost redexes of $f(f(a, b), f(b, b))$ are $a$ and $f(b, b)$. Thus

$$
f(f(a, b), f(b, b)) \rightarrow f(f(a, b), g(b))
$$

is innermost reduction, but is not

$$
f(f(a, b), f(b, b)) \rightarrow f(g(a), f(b, b)) .
$$

We will clarify the relation of two definitions of 'innermost', which are in Definition 6 and in Definition 9 , as an algorithm in Section 5 .

As suggested by Example 3, the innermost reduction is a way to construct the basic reduction sequence. The following lemma is a key result for our proof of the completeness of unification based on narrowing.

Lemma 1. Let $t$ be a term and $\eta$ be a normal substitution. If a reduction sequence

$$
t \eta \equiv t_{0} \rightarrow t_{1} \rightarrow \cdots \rightarrow t_{n}
$$

consists only of innermost reductions, then the reduction sequence is based on $O g(t)$.

Proof. The proof is by induction on the length of the reduction. Suppose first that $n=1$, and consider the narrowing sequence

$$
t \eta \equiv t_{0} \rightarrow\left[u_{0}, \alpha_{0} \rightarrow \beta, \theta_{0}\right] t_{1}
$$

Then the occurrence of every redex of $t_{0}$ is in $O g(t)$ because $\eta$ is normal. Thus the result holds by letting

$$
\begin{aligned}
& U_{0}=O g(t), \\
& U_{1}=\left(U_{0}-\left\{v \in U_{0} ; u_{0} \preceq v\right\}\right) \cup\left\{u_{0} . v ; v \in O g\left(\beta_{0}\right)\right\} .
\end{aligned}
$$

Now suppose that the result holds for $n$. Consider the sequence

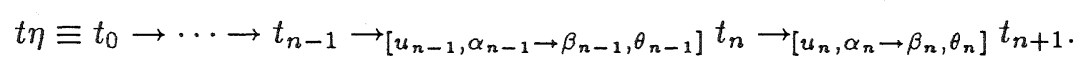

From the induction hypothesis there is a sequence of sets of occurrences $U_{0}, \ldots, U_{n}$ which satisfies the conditions $\left(\mathrm{B}-1^{\prime}\right),(\mathrm{B}-2)$, and $(\mathrm{B}-3)$ for the sequence

$$
t_{0} \rightarrow \cdots \rightarrow t_{n-1} \rightarrow t_{n}
$$

Because $t_{n-1} / u_{n-1}$ is innermost, all of its proper subterms are normal, and $\theta_{n-1}$ is also normal. Thus the occurrence of every redex of $r_{n-1} \theta_{n-1}$ is in $O g\left(r_{n-1}\right)$. So because $u_{n} \in U_{n}$, the sequence $U_{0}, \ldots, U_{n+1}$ satisfies the conditions (B-1'), (B-2), and (B-3) by letting

$$
U_{n+1}=\left(U_{n}-\left\{v \in U_{n} ; u_{n} \preceq v\right\}\right) \cup\left\{u_{n} . v ; v \in O g\left(\beta_{n}\right)\right\} \text {. }
$$

If $R$ is canonical, then $R$ has the property that there is an innermost reduction sequence from every term $t$ to $N(t)$ because any reduction sequence from $t$ reaches to $N(t)$. However the property is not confirmed in general. Therefore we need to identify the class of term rewriting systems with this property. 
Definition 10. $R$ is normalizing with innermost reductions if for every term there exists an innermost reduction to its normal form.

Then the completeness of unification based on basic narrowing is proved using the innermost reduction sequence.

Theorem 3. If $R$ is weakly canonical and normalizing with innermost reductions, then $\Sigma_{B}(t, s)$ is a complete set of $E$-unifiers of $t$ and $s$.

Proof. Let $\theta$ be an $E$-unifier of $s$ and $t$, and $\eta$ is its normal form. Because $R$ is normalizing with innermost reductions, there exists an innermost reduction sequence

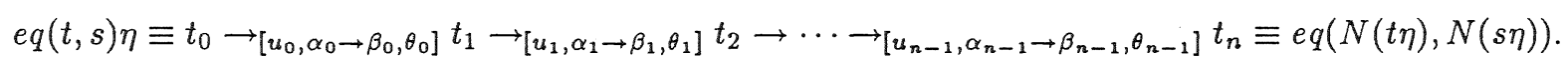

By Lemma 1 there is a sequence of sets of occurrences $U_{0}, \ldots, U_{n}$ which makes the reduction sequence basic. Because $R$ is weakly canonical, by Theorem 1 , we can construct a narrowing sequence

$$
e q(t, s) \equiv s_{0} \Rightarrow_{\left[u_{0}, \alpha_{0} \rightarrow \beta_{0}, \sigma_{0}\right]} s_{1} \Rightarrow_{\left[u_{1}, \alpha_{1} \rightarrow \beta_{1}, \sigma_{1}\right]} s_{2} \Rightarrow \cdots \Rightarrow_{\left[u_{n-1}, \alpha_{n-1} \rightarrow \beta_{n-1}, \sigma_{n-1}\right]} s_{n} \equiv e q\left(t^{\prime}, s^{\prime}\right)
$$

where $t^{\prime}$ and $s^{\prime}$ are unifiable. Thus by letting the mgu of $t^{\prime}$ and $s^{\prime}$ be $\mu$, there exists a substitution $\gamma$ such that

$$
\left.\sigma_{0} \cdots \sigma_{n-1} \mu \gamma\right|_{V(t, s)}=\left._{E} \eta\right|_{V(t, s)}
$$

The reduction sequence is based on $e q(t, s)$, there is a sequence of sets of occurrences $U_{0}, \ldots, U_{n}$. Then the narrowing sequence is also basic by using $U_{0}, \ldots, U_{n}$ because the two sequence use the same occurrence at each step. Moreover, $t^{\prime}$ and $s^{\prime}$ are unifiable, and there is a substitution $\gamma$ such that $t_{n}$ and $s_{n}$ are unifiable with the mgu $\mu$ and

$$
\left.\sigma_{0} \cdots \sigma_{n-1} \mu \gamma\right|_{V(t, s)}=\left.{ }_{E} \eta\right|_{V(t, s)}
$$

by Theorem 1 .

If $R$ is canonical, then $R$ is weakly canonical and normalizing with innermost reductions because every reduction from a term $t$ reaches to $N(t)$. Thus Theorem 3 holds for canonical term rewriting systems. 


\section{Switching Lemma for Basic Narrowing}

In this section we discuss the relation between Theorem 3 and Algorithm 1. The most important point of Algorithm 1 is the computation rule $S$. Thus we give the switching lemma that confirms the introduction of $S$ into basic narrowing as in the theory of SLD-resolution.

At first we consider a procedure for innermost reduction. The following Algorithm 2 is the representation.

\section{Algorithm 2.}

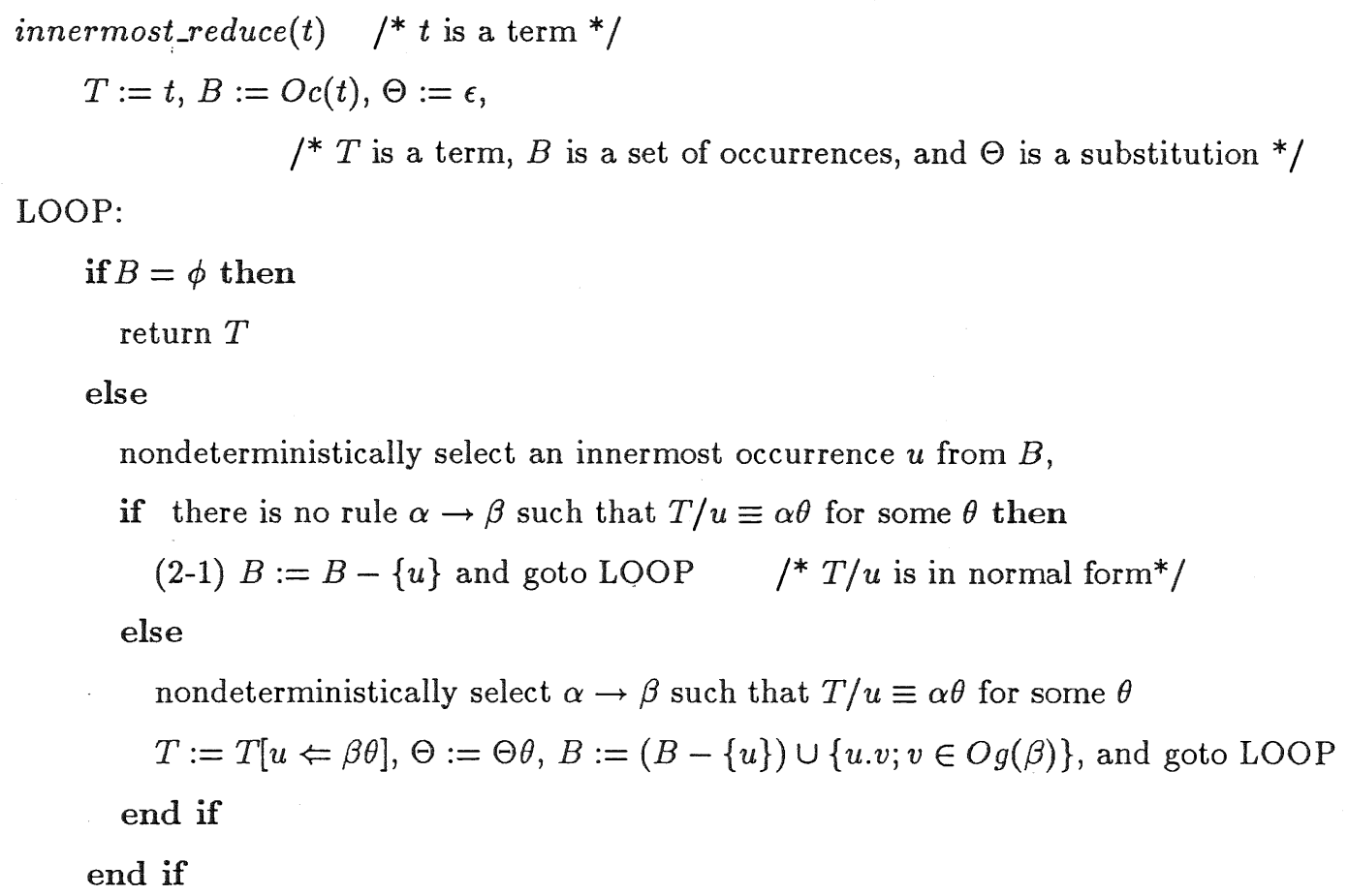

In Algorithm 2 the procedure which searches an innermost redex from a term is clarified by using the set $B$ and the step (2-1). The step shows the difference between Definition 6 and Definition 9.

Now we apply Algorithm 2 to the construction of the basic reduction sequence in the proof of Theorem 3 . The reduction sequence and the basic narrowing sequence in the proof use the same sequence $U_{0}, \ldots, U_{n}$. Thus it is clear that we may add a step corresponding to (2-1) to the procedure for basic narrowing. In Algorithm 1 the step is (1-1).

Then in order to prove the completeness of Algorithm 1 using Theorem 3, we need to show that we may restrict the selection of innermost occurrences with a computation rule $S$. This restriction is allowable because of the following switching lemma for Algorithm 1. To explain the lemma, in Algorithm 1, we use symbols $E(S, n), B(S, n), \Theta(S, n)$ to represent the values of $E, B$, and $\Theta$ at the beginning of the $n$-th execution of the LOOP when the computation rule is $S$.

Lemma 2. Suppose that there are two innermost occurrences $u$ and $v$ in $B(S, n)$ and that $S$ selects $u$ in the $n$-th execution of the LOOP and $v$ in the $(n+1)$-st. Then we may use the computation rule $S^{\prime}$ which selects $v$ instead of $u$ at the $n$-th execution of LOOP and $u$ at the $(n+1)$-st. Moreover we can let $E(S, n+2)$ and $E\left(S^{\prime}, n+2\right)$ be variants, $\Theta(S, n)$ and $\Theta\left(S^{\prime}, n\right)$ be variants, and $B(S, n+2)=B\left(S^{\prime}, n+2\right)$. 
It should be noticed that Lemma 2 is proved by translating the basic narrowing sequence to SLD-refutation and applying the switching lemma for SLD-refutation in [1]. Of course, the false assertion was used in [1]. Thus we omit its proof.

\section{Conclusion}

We have proved the completeness of unification based on narrowing in a weaker restriction and have proved the completeness of unification based on basic narrowing, and have given the switching lemma. The discussion in [1] is important but was only a half way to the completeness because it showed only the switching lemma.

In the discussions above, we have used the conditions that $R$ is weakly canonical and is normalizing with innermost reductions. Both [1] and [2] treat the case that $R$ is a canonical term rewriting system, which is useful for the theorem provers using reduction. In the discussions on narrowing, the restriction for term rewriting systems should be weakened because unification based on narrowing is a refutation procedure. We have shown that they can be really weakened.

We need to find the sufficient conditions on which a term rewriting system is weakly canonical and normalizing with innermost reductions. Especially, we have not clarified any properties of term rewriting systems which is normalizing with innermost reductions. We conjecture that weakly canonical term rewriting systems are normalizing with innermost reductions.

We need also to import the property of SLD-resolution other than the switching lemma into the theory of narrowing. For example, we are considering the complete definitions of functions in the same way as those of predicates in SLD-resolution.

\section{Acknowledgements}

The author would like to thank Prof. Setsuo Arikawa for his constructive comments and encouragements. The author also wishes to thank Dr. Makoto Haraguchi for many discussions on narrowing and SLD-resolution.

\section{References}

[1] Bosco, P.G., Giovannetti, E., and Moiso, C. (1987): Refined Strategies for Semantic Unification, Proc. TAPSOFT' 87 (LNCS 250), 276-290.

[2] Hullot, J.M. (1980): Canonical Forms and Unification, Proc. 5th Conference on Automated Deduction (LNCS 87), 318-334.

[3] Lloyd, J.W. (1984): Foundations of Logic Programming, Springer-Verlag.

[4] Martelli, A. and Montanari, U. (1982): An Efficient Unification Algorithm, ACM TOPLAS, 4(2), 258-282.

[5] Yamamoto, A. (1987): A Theoretical Combination of SLD-Resolution and Narrowing, Proc. 4th ICLP, $470-487$. 